This is an Accepted Manuscript of an article published by Taylor \& Francis in Cognition \& Emotion in 2020, available online: https://doi.org/10.1080/02699931.2019.1675596

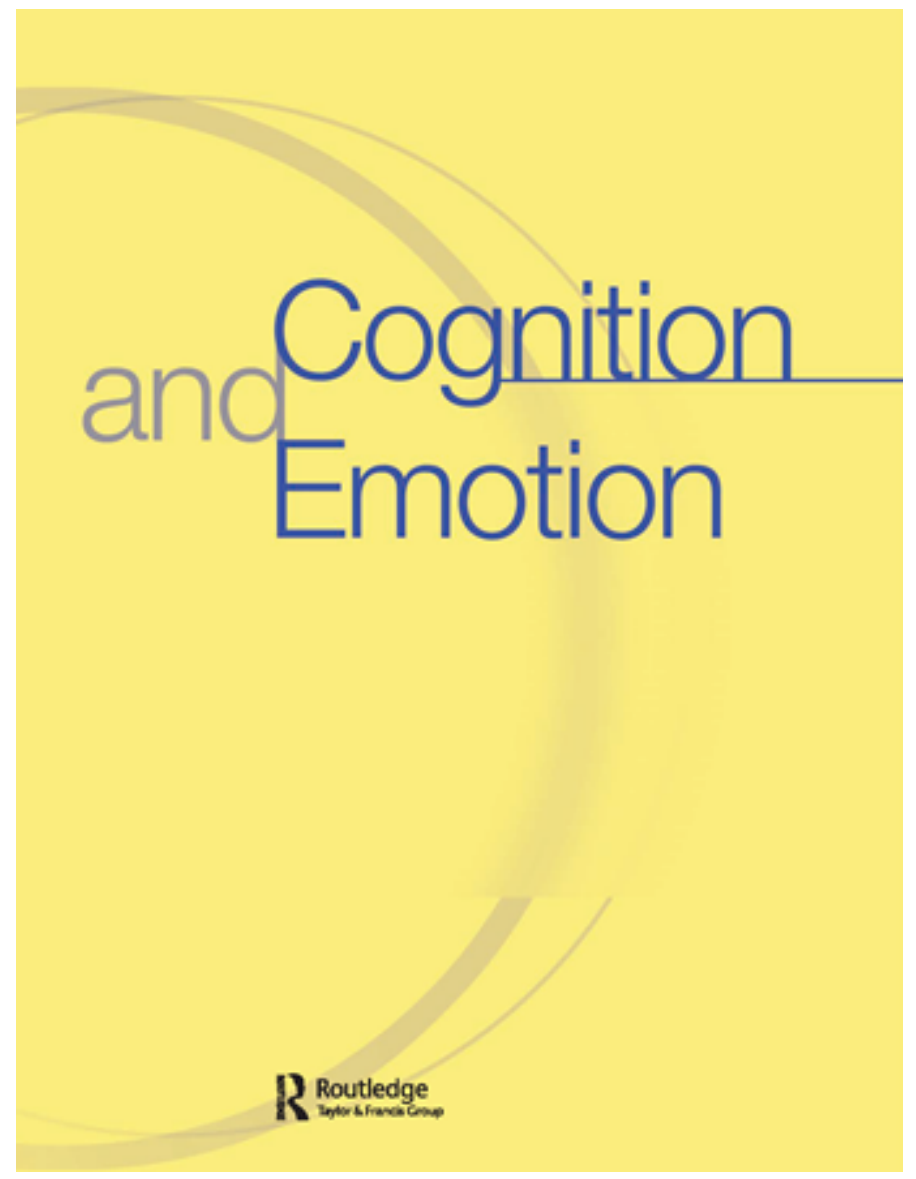

\title{
Social Anxiety is Associated with Impaired Memory for Imagined Social Events with Positive Outcomes
}

\begin{tabular}{|c|c|}
\hline Journal: & Cognition and Emotion \\
\hline Manuscript ID & Draft \\
\hline Manuscript Type: & Full Article \\
\hline $\begin{array}{r}\text { Date Submitted by the } \\
\text { Author: }\end{array}$ & $n / a$ \\
\hline Complete List of Authors: & $\begin{array}{l}\text { Romano, Mia; University of Waterloo, University of Waterloo } \\
\text { Tran, Emma; University of Waterloo, University of Waterloo } \\
\text { Moscovitch, David; University of Waterloo, University of Waterloo }\end{array}$ \\
\hline Keywords: & $\begin{array}{l}\text { social anxiety, memory bias, positivity deficit, episodic memory, } \\
\text { imagined scenarios }\end{array}$ \\
\hline
\end{tabular}




\section{SCHOLARONE ${ }^{\text {W }}$ Manuscripts}




\title{
MEMORY BIASES IN SOCIAL ANXIETY
}

\section{Social Anxiety is Associated with Impaired Memory for Imagined Social Events with}

\section{Positive Outcomes}

\author{
Mia Romano, Emma Tran, \& David A. Moscovitch
}

Department of Psychology and Centre for Mental Health Research and Treatment, University of Waterloo

\footnotetext{
Corresponding author: David A. Moscovitch, Department of Psychology and Centre for Mental Health Research and Treatment, University of Waterloo; Waterloo, Ontario, Canada N2L-3G1. Contact: dmosco@uwaterloo.ca.
} 


\title{
MEMORY BIASES IN SOCIAL ANXIETY
}

\begin{abstract}
Cognitive models of social anxiety disorder suggest that memory biases for negative social information contribute to symptoms of social anxiety (SA). However, it remains unclear whether memory biases in SA are related to social information, specifically, and if so, whether the valence of such information would moderate memory performance. In the present study, 197 community participants were randomised to imagine themselves as the central character in either 10 social or 10 non-social scenarios. In both conditions, half of the scenarios ended with objectively positive outcomes and half ended with objectively negative outcomes. Results demonstrated that higher trait SA was related to memory performance for social scenarios only, and in particular to poorer memory for social scenarios that ended positively. Thus, the impact of SA on memory performance depended on how social information was framed, with higher SA related to poorer memory for positive social experiences. These context-specific effects contribute to the growing literature on positivity deficits in SA.
\end{abstract}

Keywords: social anxiety, memory bias, positivity deficit, episodic memory, imagined scenarios 


\section{MEMORY BIASES IN SOCIAL ANXIETY}

\section{Introduction}

High socially anxious (SA) individuals, including those with social anxiety disorder (SAD), are preoccupied with the possibility of scrutiny and evaluation in anticipation of and during social interaction (American Psychiatric Association, 2013). They have a persistent fear of situations where they may be evaluated by others - fear which research has shown is often driven by cognitive biases that impact their perceptions of themselves and others within socially threatening contexts (Clark \& McManus, 2002).

According to cognitive models of SA (Clark \& Wells, 1995; Rapee \& Heimberg, 1997), socially anxious individuals base their perceptions and actions upon negatively distorted mental self-images that developed from prior social experiences and are represented in long-term memory in a negatively-biased manner (Hackmann, Clark, \& McManus, 2000; Hackmann, Suraway, \& Clark, 1998). These negatively-biased memories are selectively retrieved by high SA individuals within anxiety-provoking social contexts, and can inform expectations and appraisals of current social and interpersonal demands (Clark, 1999; Mathews \& Macleod, 2005). For example, in anticipation of giving a work presentation, a socially anxious individual who worries about appearing awkward may spontaneously and selectively recollect past negative experiences in which she felt awkward or behaved in a manner she perceived as being awkward, as well as general semantic knowledge or self-schema related to being "an awkward person" that is derived from such experiences. This bias toward retrieval of negative information from memory serves to increase anxiety and apprehension during social situations (Hirsch \& Clark, 2004) and leads to the exacerbation of negative beliefs as well as maladaptive emotional responses and avoidance behaviours (Clark, 1999; Mathews \& MacLeod, 2005; Romano, Ma, Moscovitch, \& Moscovitch, in press). 


\section{MEMORY BIASES IN SOCIAL ANXIETY}

Given their preferential retrieval of negative social memories, do high SA individuals tend to show memory biases for negative social information? Though the literature on episodic memory biases in SA is characterised by varied methodological approaches (Morgan, 2010; Zlomuzica et al., 2014), some studies have found that high SA individuals exhibit enhanced memory for social threat information, such as negative words or critical facial expressions (e.g., Lundh \& Öst, 1996; Mansell \& Clark, 1999). Similarly, studies of memory for autobiographical past events have demonstrated that higher trait SA is associated with elevated retrieval of threatening and highly emotional material (Krans, de Bree, \& Bryant, 2013; Moscovitch et al., 2018; Wenzel \& Cochran, 2006).

Moscovitch et al. (2018) found that while participants with SAD and healthy controls could retrieve autobiographical memories of both aversive and non-aversive social experiences with similar ease, SAD participants' memories of negative events contained greater episodic detail (i.e., more specific information about those particular negative experiences) than memories of negative events that were retrieved by controls; however, there were no such differences between groups for memories of non-aversive past events. It is unclear, however, whether these findings suggest that those with SAD have superior memory for the details of past negative events or whether they tend to embellish their memories with details that are consistent with their negative self-schemas but may not, in fact, be accurate (e.g., Hertel, Brozovich, Joorman, \& Gotlib, 2008; Hirsch, Clark, \& Mathews, 2006). Unfortunately, studies of autobiographical memories of personal past events rarely measure the accuracy of participants' reported memories, as doing so would require an objective record of the event against which participant reports could be compared and verified. Previous work has demonstrated that socially anxious individuals' interpretations of ambiguous social scenarios during encoding often emerge during 


\section{MEMORY BIASES IN SOCIAL ANXIETY}

later recall as negatively-biased memory intrusions which they erroneously assume to be accurate (Hertel et al., 2008). Hertel et al.'s findings suggest that episodic memory enhancements for negative social material may represent intrusions or embellishments of false but schemaconsistent information rather than enhanced but accurate memory for past negative events, though additional research is needed to support this conclusion.

Another issue requiring empirical clarification is whether enhanced memory for negative social information among high SA individuals might also extend to negative non-social information. Given that individuals with higher levels of SA are prone to negative affect (Brown, Chorpita, \& Barlow, 1998), they may lack the self-protective tendency to recall events in an adaptive manner (e.g., Koban et al., 2017; Wilson \& Ross, 2003), which may result in better memory for negative emotional material of any sort, even beyond social contexts. However, while Hertel and colleagues (2008) demonstrated that participants with SAD showed negative interpretations and biased recall for ambiguous social scenarios, non-social scenarios did not lend themselves to threatening interpretations or biased recall, which may suggest that such biases occur only for social events. Thus, another aim of our study was to examine whether enhanced memory recall of negative information among higher SA individuals encompasses any negative emotional material or is specific to negative social information.

A final issue requiring clarification, which we aimed to investigate in the present study, is whether the effects of SA on memory biases might also apply to positive social or non-social information. Non-anxious individuals often demonstrate a positivity bias that aligns with current self-appraisals, recalling events as more positive than they were, irrespective of whether they were social or non-social in nature (e.g., Levine, Schmidt, Kang, \& Tinti, 2012; Schacter, Guerin, \& St. Jacques, 2011; Wilson \& Ross, 2003). A growing body of research has 


\section{MEMORY BIASES IN SOCIAL ANXIETY}

documented a variety of "positivity deficits" for people with higher levels of SA across a range of situations and experiences (Kashdan, 2007; Kashdan, Weeks, \& Savostyanova, 2011). To this end, individuals with high SA or SAD have been shown to underestimate their social competence even in positive interactions (Kashdan, Morina, \& Priebe, 2009), report fewer and less intense positive emotions in response to social experiences (Kashdan \& Collins, 2010), endorse fewer everyday positive experiences relative to controls (Kashdan \& Steger, 2006), and demonstrate reduced capacity to savour positive experiences of any nature (Eisner, Johnson, \& Carver, 2009). Thus, it may be reasonable to predict that higher levels of SA would be associated with deficits in the recollection of positive social memories. However, Moscovitch et al. (2018) found no differences between SAD and healthy control participants' recollection of non-negative social events about which participants reported little anxiety, though their study did not examine memory for objectively positive events.

Consistent with the positivity deficit hypothesis, recent studies have found that both high levels of SA and a clinical diagnosis of SAD predict the erosion of memory for positive feedback about social performance (Glazier \& Alden, 2017, 2019). In these studies, socially anxious and non-anxious participants recalled positive or neutral feedback they received after performing a social task. There were no group differences in recall when memory performance was measured 5 minutes after the task; however, at one week delay, socially anxious participants recalled signficantly fewer positive details than non-anxious controls, a difference that did not extend to memory for neutral feedback. These findings suggest that SA may erode memory for positive elements of social events, which warrants further investigation.

No studies to our knowledge have examined the relation between trait SA and memory for objectively positive events, such as interpersonal successes or social achievements. 


\section{MEMORY BIASES IN SOCIAL ANXIETY}

Therefore, a final aim was to examine whether high levels of trait SA might be associated with deficits in memory retrieval for positive social memories.

\section{The present study}

This study examined the conditions under which episodic memory biases might arise for individuals with higher levels of SA. Community participants across the SA spectrum were randomised to imagine themselves as the central character in 10 social or non-social scenarios. In both conditions, half of the scenarios ended with objectively positive outcomes and half ended with objectively negative outcomes. Participants were then required to recall or recognise various details of each scenario. We hypothesised that higher levels of trait SA would predict superior memory for negatively-valenced social scenarios and diminished memory for positively-valenced social scenarios, but that SA would not be related to memory outcomes for non-social scenarios. We tested these hypotheses by examining participant performance on three memory tasks: (a) free recall of the scenario titles (i.e., themes); (b) recall of the valence of scenario outcomes as positive or negative; and (c) recognition of neutrally-valenced scenario details. For memory task 1 (free recall of scenario titles), we expected to observe an interactive effect of trait SA and scenario context condition, such that higher levels of SA would predict greater recall of social scenario titles for scenarios ending with negative outcomes and fewer titles for scenarios ending with positive outcomes, but that the effect of SA would not be present for non-social scenarios. For memory task 2 (recall of whether the valence of scenario outcomes was positive or negative), we expected that higher levels of SA would predict greater accuracy of recall for the valence of outcomes of social scenarios that ended negatively and reduced accuracy for scenarios that ended positively, but no effects of SA for non-social scenarios regardless of outcome valence. For memory task 3 (recognition of neutrally-valenced scenario details), we 


\section{MEMORY BIASES IN SOCIAL ANXIETY}

expected that higher levels of SA would be related to more accurate recognition for neutral details of negatively-valenced social scenarios and poorer recognition for neutral details of positively-valenced social scenarios; again, we expected a significant interaction, whereby levels of SA would only predict recognition of neutral details in the social condition but not in the nonsocial condition.
Method
Participants
A total of 243 community participants were sampled from Amazon Mechanical Turk (MTurk). Participants were required to: (a) be living in Canada or the US, (b) meet technical requirements for completing the online study (e.g., endorsed having access to a secure laptop that could play audio files), and (c) have a completion rate of $95 \%$ from participation in past MTurk studies.

\section{Measures}

Social Interaction Anxiety Scale (SIAS; Mattick \& Clarke, 1998). The SIAS is a commonly used 20-item self-report questionnaire for assessing symptoms of SA. Respondents indicate the degree to which 20 statements about anxiety in social interaction situations feel characteristic of them on a 5-point Likert-type scale (ranging from $0=$ Not at all characteristic or true of me to $4=$ Extremely characteristic or true of me). The SIAS was administered as part of a larger questionnaire battery, completed by participants after the memory recall tasks $(\alpha=$ $.92)$.

\section{Depression Subscale of the Depression Anxiety Stress Scales - 21-Item Version}

(DASS-21; Lovibond \& Lovibond, 1995). The DASS-21 is a short version of the 42-item Depression Anxiety Stress Scales (DASS; Lovibond \& Lovibond, 1995), which measures 


\section{MEMORY BIASES IN SOCIAL ANXIETY}

depression, anxiety, and stress. The present study used the 7-item depression subscale of the DASS-21 to assess depression symptoms. Participants self-reported the degree to which seven statements applied to them over the past week on a 4-point Likert-type scale (ranging from $0=$ Did not apply to me at all to $3=$ Applied to me very much or most of the time). The depression subscale of the DASS-21 was administered to participants after the memory recall tasks, and was included to ensure that participants' depression symptoms were equivalent across the two conditions $(\alpha=.94)$.

Vividness of Visual Imagery Questionnaire - Short Version (VVIQ-Short; Reisberg, Pearson, \& Kosslyn, 2003). The VVIQ-Short is the 4-item version of the VVIQ (Marks, 1973), a self-report questionnaire which assesses the extent to which respondents report being able to form clear and vivid images in their minds. Participants are instructed to "visualise a rising sun" and then respond to four items regarding the vividness of their mental imagery of that scene on a 5-point Likert-type scale (ranging from $1=$ Perfectly clear and as vivid as normal vision to $5=$ No image at all, you only "know" that you are thinking of the object). The VVIQ-Short was administered to participants after the memory recall tasks, and was included to establish that participants across conditions did not differ in their ability to form clear mental images, as participants were instructed to imagine themselves as the lead character within the scenarios presented $(\alpha=.86)$.

Data quality check questions. A set of quality and engagement check questions was administered at the end of the study in order to ensure participants were paying attention (e.g., "As you were going through this psychology study, did you develop any ideas about what we might be studying?"), and to request that they self-identify any issues that arose during the study that may have compromised the validity of their data ("Should we use your data in our 


\section{MEMORY BIASES IN SOCIAL ANXIETY}

analysis?"). As noted below, some participants were excluded for reporting invalid data due to technical issues they experienced with the presentation of study stimuli.

\section{Scenario Stimuli}

In each of the two scenario context conditions, participants were presented, one at a time, with 10 scenarios, worded in the present tense, for which they were asked to imagine themselves as the actor or main character. The instructions encouraged participants to fully immerse themselves in the scenarios by imagining the scenarios from a first-person perspective as vividly as possible, as if all the events were happening to them. Each scenario was presented simultaneously in visual and audio formats, with words both written on screen and read slowly aloud in a clear, pre-recorded voice. The scenarios were constructed in a standardised manner to contain an equivalent amount of information; thus, the length of each of the audio recordings was 25 to 30 seconds long across all stimuli. The scenarios were adapted from ambiguous scenarios used in past studies of cognitive biases in SA (Amin, Foa, \& Coles, 1998; Berna, Lang, Goodwin, \& Holmes, 2011; Hertel et al., 2008). All participants were randomly assigned to receive either social or non-social scenarios. Within each condition, half of the 10 scenarios were randomly assigned a positive outcome and the other half were negative. The order of presentation of the 10 scenarios differed for every participant. As the study was administered online, instructions pertaining to scenario stimuli emphasised the importance of completing the task in an environment with minimal distractions. Participants were instructed to pay as close attention as possible to each stimulus and were prohibited from taking any notes.

\section{Scenario Structure and Composition}

Title and first sentence. All scenarios were preceded by a short title briefly describing the key event. The title appeared alone on screen for three seconds before advancing 


\section{MEMORY BIASES IN SOCIAL ANXIETY}

automatically to the next screen containing the first sentence. The first sentence always framed the scenario as being either social or non-social but contained no information about whether the outcome was negative or positive (i.e., its valence remained ambiguous). Social scenarios were described as involving social aspects at their core, where social evaluation by others was a possibility. For example, the first sentence of a social scenario entitled "Blind Date" was: You go on a blind date. In contrast, a non-social scenario was not social at its core, such that the key event involved the person acting alone or did not primarily concern others. For example, the first sentence of a non-social scenario entitled "The Wallet" was: You reach for your wallet as you leave the house.

Middle steps. Following the presentation of the scenario title and ambiguously-valenced first sentence, the screen advanced to the middle steps of the scenario, which were always provided in three brief sentences. Like the first sentence, the middle steps were emotionally neutral regardless of the scenario's outcome, meaning that they provided details devoid of valenced information that would reflect the outcome of the scenario. Regardless of whether the eventual outcome was positive or negative, the middle steps simply described objective events that unfolded in the scenario in a manner that was consistent with the context condition for each scenario. For example, the middle steps for the scenario "Blind Date" were: You greet your date with a handshake. You each talk about your hobbies. You order food and drinks.

Final sentence. Following the neutral middle details, the screen advanced a final time to reveal an objectively positive or negative outcome, which disambiguated the valence of the scenario and identified the central character's experience as being either a success or a failure. An objectively positive outcome was one where the actor in the scenario achieved or succeeded at something meaningful, whereas a negative outcome involved being unsuccessful or failing to 


\section{MEMORY BIASES IN SOCIAL ANXIETY}

achieve something meaningful. For example, the following were the positive and negative outcomes for the "Blind Date" and "The Wallet" scenarios:

Blind Date:

Positive outcome: When the date comes to an end, your date tells you they are interested in seeing you again.

Negative outcome: When the date comes to an end, your date tells you they are not interested in seeing you again.

The Wallet:

Positive outcome: You grab your wallet and head out the door.

Negative outcome: You can't find your wallet and head out the door empty-handed.

\section{Memory Tasks}

Immediately following the presentation of the 10 scenarios, participants were given three surprise memory tasks for the encoded material.

\section{Memory task 1: free recall of scenario titles}

Free recall of scenario titles. First, participants were asked to recall as many scenario titles (e.g., Blind Date, The Wallet) as they could in an open-ended response box. Of interest for data analyses was the number of scenario titles accurately recalled between scenario context conditions (social vs. non-social) and across valenced outcomes (positive vs. negative), as a function of trait SA.

Scoring procedure. Participants scored 1 point for each scenario they accurately recalled. Keywords and themes of the scenarios were considered acceptable responses - for example, both "drama" and "play" were considered correct for the scenario "Drama Group" involving a community performance. A score out of 5 was calculated for each outcome valence. 


\section{MEMORY BIASES IN SOCIAL ANXIETY}

\section{Memory task 2: recall of outcome valence}

Recall of outcome valence as positive or negative. Participants were then provided with

the title and first line of all scenarios they encoded, one at a time, and asked to recall whether the outcome for each was positive or negative. Of interest for data analyses was the number of scenario outcomes accurately recalled as being either positive or negative between conditions and across valenced outcomes, as a function of trait SA.

Scoring procedure. For each outcome correctly identified as either positive or negative, participants were given a score of 1 , for a total of 5 per outcome valence. For instance, for positively-valenced scenarios, a score of 5 would indicate that the participant accurately recalled that all five positively-valenced scenarios ended positively, while a score of 0 would indicate that the participant inaccurately identified all the scenarios' outcomes as negative.

\section{Memory task 3: recognition of middle steps}

Recognition of middle steps as old or new. Finally, participants completed a recognition memory task. For each scenario that participants had imagined, the title and first line were provided in addition to two middle details - one of which had been presented in the original scenario ("old"), and the other had not ("new"). For each of the 10 scenarios, participants were tasked with deciding whether each of the two middle details presented was old or new, for a total of 20 questions in this task. For example, the following were the test items for the "Blind Date" and "The Wallet" scenarios:

Blind Date:

You go on a blind date...

a) You each talk about your hobbies. (old)

b) You ask your date about their work. (new) 


\section{MEMORY BIASES IN SOCIAL ANXIETY}

The Wallet:

You reach for your wallet as you leave the house...

a) You put on your jacket. (old)

b) You check all your drawers. (new)

Scoring procedure. To examine recognition memory, four different scores were calculated for each scenario across outcome valence, which represented the match/mismatch between whether the detail was old or new, and whether the detail was identified by participants as old or new: hit rate (actually old and identified as old), miss rate (actually old, identified as new), correct rejection rate (actually new, identified as new), and false alarm rate (actually new, identified as old). Given that we were interested in actual memory for previously encountered stimuli and that hit and miss scores represent the inverse of one another, analyses pertained to only hit scores. Participants could receive a maximum score of 1 per scenario for a total of 5 per outcome valence. A hit score of 5 (corresponding with a miss score of 0 ) would indicate that the participant correctly recalled all the old details as "old."

\section{Procedure}

The study was hosted online via Qualtrics. After completing a demographics questionnaire participants were introduced to the encoding phase of the study, in which they saw scenarios they were instructed to imagine and visualise themselves experiencing from a firstperson perspective. Participants first completed a practice trial with two scenarios. Then, participants were randomly assigned to imagine either 10 social or non-social scenarios. Each scenario stimulus consisted of four parts, presented one at a time and in the following order: the title of the scenario, its ambiguously-valenced first sentence (which framed the scenario as social or non-social depending on the condition to which participants were assigned), the neutral 


\title{
MEMORY BIASES IN SOCIAL ANXIETY
}

middle steps bridging the first sentence with the outcome, and the randomised valenced outcome (either positive or negative). For each part, content was presented simultaneously both as text on screen and as an audio-recording. Following the scenarios, participants completed three surprise memory tasks by typing their responses on screen. Finally, they completed a set of questionnaires: the SIAS, the DASS-21, the VVIQ-Short, and the data quality check questions, which were presented in the same order for every participant. The procedural flow is depicted in Figure 1 . The total study length was approximately 40 minutes, and participants were remunerated \$1.50 USD toward their MTurk accounts for their participation. This study was reviewed and received ethics clearance through the research ethics board at the authors' institution.

\begin{abstract}
Results
Preliminary analyses

Participant characteristics. From the 243 participants recruited from MTurk, 46 were excluded from analyses: one was excluded for not giving post-debriefing consent, six were identifiable robots, 28 gave unintelligible responses throughout the study, one did not complete any memory tasks, and 10 reported technical difficulty with the audio files. The final study sample for data analysis, therefore, consisted of 197 participants (98 in the social condition and 99 in the non-social condition). Of the 197 participants, there was missing data pertaining to one participant's age (social condition) and two participants' SIAS scores (non-social condition).
\end{abstract}

Equivalence of conditions. As shown in Table 1, there were no significant differences between participants across the social and non-social conditions in demographic characteristics or in responses to symptom measures (SIAS, DASS) or imagery ability (VVIQ-Short). Main analyses 


\section{MEMORY BIASES IN SOCIAL ANXIETY}

The study was based on a 2x2 mixed multifactorial design. The between-subjects factor was scenario context condition (social vs. non-social), and the within-subjects factor was scenario outcome valence (negative vs. positive). Trait SA was used as a continuous predictor of the three different memory outcomes for positive and negative social scenarios separately.

The data for all memory tasks were analysed using hierarchical linear regressions with SIAS scores and scenario context condition (social vs. non-social) entered in the first step and the interaction between these two variables entered into the second step. Positive and negative memory outcomes for each memory task were entered as the dependent variable in each regression model, with separate regression analyses conducted for positive memory outcomes and then negative memory outcomes. Significant interactions were probed by running separate regression analyses for the social and non-social conditions in which we examined the influence of SIAS scores on each condition in turn.

\section{Memory Task 1: Free Recall of Scenario Titles}

Positive outcomes: Step 1 of the hierarchical regression model was significant $\left(F_{(2,192)}\right.$

$=4.24, p=.016)$, accounting for $4.2 \%$ of the overall variance in free recall of positive outcome scenario titles. The SIAS x condition interaction term was entered into the second step of the regression model. When including the interaction term, the model was statistically significant $\left(F_{(3,191)}=4.35, p=.005\right)$ and accounted for $2.2 \%$ additional variance $\left(\Delta \mathrm{R}^{2}=.022 ; \Delta F_{(1,191)}=4.43, p=.037\right)$. Parameter estimates in the second step indicated a significant main effect of SIAS, where higher scores predicted worse 
MEMORY BIASES IN SOCIAL ANXIETY

memory performance $(\beta=-0.30, t=-3.02, p=.003)$, a non-significant effect of condition $(\beta=$

$-0.13, t=-1.89, p=.062)$, and a significant interaction between SIAS and condition $(\beta=0.21, t$

$=2.11, p=.037)$. To probe the nature of the interaction we first examined the effect of SIAS in the social condition. The results demonstrated that higher SIAS scores significantly predicted poorer recall of positive scenario outcome titles $\left(F_{(1,96)}=9.43, \beta=-0.30, t=-3.07, p=.003\right)$, accounting for $8.9 \%$ of the variance. In the non-social condition, however, SIAS scores did not significantly predict memory for outcome titles $\left(F_{(1,95)}=0.00, \beta=-0.00, t=-0.02, p=.981\right)$.

Negative outcomes: In the model examining the effects of SIAS, condition, and their interaction predicting free recall of scenario titles for scenarios ending negatively, steps 1 and 2 were both non-significant $(F ' s<1.26, p$ 's $>.290)$.

\section{Memory Task 2: Recall of Outcome Valence}

Positive outcomes: Step 1 of the hierarchical regression model was significant $\left(F_{(2,192)}\right.$

$=7.25, p=.001)$ and accounted for $7.0 \%$ of the variance in the correct recall of positive scenario outcome valence. The SIAS x condition interaction term was entered into the second step of the regression model. This model was statistically significant $\left(F_{(3,191)}=\right.$ $6.04, p=.001)$ and accounted for $1.6 \%$ additional variance, although the change in $\mathrm{R}^{2}$ was not significant $\left(\Delta \mathrm{R}^{2}=.016 ; \Delta F_{(1,191)}=3.43, p=.066\right)$. Parameter estimates in the second step indicated a significant main effect of SIAS where higher scores predicted 


\section{MEMORY BIASES IN SOCIAL ANXIETY}

poorer memory performance $(\beta=-0.39, t=-4.00, p<.001)$, a non-significant effect of condition $(\beta=-0.03, t=-0.42, p=.678)$ and a marginally significant interaction between SIAS and condition $(\beta=0.18, t=1.85, p=.066)^{1}$.

Negative outcomes: In the model predicting the correct recall of scenario outcomes for scenarios ending negatively, step 1 was significant $\left(F_{(2,192)}=3.44, p=.034\right)$, and accounted for $3.5 \%$ of the variance. While the overall model for step 2 was significant $\left(F_{(3,191)}=2.70, p=\right.$ $.047)$, the addition of the interaction term was non-significant $\left(\Delta \mathrm{R}^{2}=.006 ; \Delta F_{(1,191)}=1.21, p=\right.$ .273). Parameter estimates in the second step indicated a significant effect of condition $(\beta=$ $0.16, t=2.24, p=.027)$, where the non-social condition was related to better memory for scenario outcomes; however, SIAS scores did not predict significant variance in memory for scenario outcomes $(\beta=-0.18, t=-1.82, p=.070)$, nor did the interaction between SIAS scores and condition $(\beta=0.11, t=1.10, p=.273)$.

\section{Memory Task 3: Recognition of Middle Steps}

Hit rate for positive scenarios. Step 1 of the hierarchical regression model was

significant $\left(F_{(2,192)}=3.70, p=.026\right)$ and accounted for $3.7 \%$ of the variance towards

\footnotetext{
${ }^{1}$ Although the interaction did not quite reach the required threshold for statistical significance, we nonetheless assessed whether the nature of the interaction was consistent with the results for memory task 1 . We therefore probed the nature of the interaction by first examining the effect of SIAS in the social condition. The results demonstrated that SIAS scores were a significant predictor of the recall of positive scenario outcomes $(\beta=-0.41, t=$ $-4.41, p<.001)$, accounting for $16.8 \%$ of the variance. In the non-social condition, SIAS scores did not significantly predict memory for positive outcomes $(\beta=-0.13, t=-1.23, p=.221)$.
} 
MEMORY BIASES IN SOCIAL ANXIETY

correct identification of middles steps for positive scenarios. The SIAS x condition

interaction term was entered into the second step of the regression model. Step 2 was

significant $\left(F_{(3,191)}=4.23, p=.006\right)$ and accounted for $2.5 \%$ additional variance $\left(\Delta \mathrm{R}^{2}=\right.$

$\left..025 ; \Delta F_{(1,191)}=5.12, p=.025\right)$. Parameter estimates in the second step indicated a

significant main effect of SIAS, where higher SIAS scores predicted poorer memory

performance $(\beta=-0.33, t=-3.31, p=.001)$, a non-significant main effect of condition $(\beta=$

$0.10, t=1.41, p=.161)$, and a significant interaction between SIAS and condition $(\beta=0.22, t=$

$2.26, p=.025)$. To probe the nature of the interaction, we first examined the effect of SIAS in the social condition. The results demonstrated that higher SIAS scores significantly predicted poorer recognition of middle steps for positive social scenarios $\left(F_{(1,96)}=10.73, \beta=-0.32, t=-3.28, p\right.$ $=.001$ ), accounting for $10.1 \%$ of the variance. For non-social scenarios, however, SIAS scores did not significantly predict recognition of middle steps for positive scenarios $\left(F_{(1,95)}=0.01, \beta\right.$ $=-0.01, t=-0.09, p=.929)$.

Hit rate for negative scenarios. Step 1 of the hierarchical regression model was nonsignificant $\left(F_{(2,192)}=1.95, p=.146\right)$, accounting for only $2.0 \%$ of the variance towards correct identification of middle steps for negative scenarios. The SIAS x condition interaction term was entered into the second step of the regression model. This model 
MEMORY BIASES IN SOCIAL ANXIETY

was statistically significant $\left(F_{(3,191)}=2.91, p=.036\right)$ and accounted for $2.4 \%$ additional

variance $\left(\Delta \mathrm{R}^{2}=.024 ; \Delta F_{(1,191)}=4.75, p=.030\right)$. Parameter estimates in the second step

indicated a significant main effect of SIAS where higher SIAS scores predicted poorer

memory performance $(\beta=-0.23, t=-2.33, p=.021)$, a non-significant main effect of condition

$(\beta=0.12, t=1.69, p=.092)$, as well as a significant interaction between SIAS and condition $(\beta$

$=0.22, t=2.18, p=.030)$. To probe the nature of the interaction we first examined the effect of

SIAS in the social condition. Mirroring results for positive scenarios, results demonstrated that higher SIAS scores significantly predicted poorer recognition of middle steps for negative scenarios $\left(F_{(1,96)}=5.32, \beta=-0.23, t=-2.31, p=.023\right)$, accounting for $5.2 \%$ of the variance. In the non-social condition, however, SIAS scores did not significantly predict recognition of middle steps for negative scenarios $\left(F_{(1,95)}=0.60, \beta=0.08, t=0.77, p=.442\right)$.

\section{Discussion}

In the present study, we investigated the effects of trait SA and social context on episodic memory performance for positively and negatively-valenced scenarios that were encoded by participants who imagined themselves as the central character. We predicted that participants with higher levels of SA would demonstrate superior memory performance for negativelyvalenced scenarios and poorer memory for positively-valenced scenarios, but that these effects would only emerge when scenarios were embedded within a social context. We expected similar patterns of performance for each of the three memory tasks: recalling the titles of encoded scenarios, recalling their outcome valence, and correctly identifying their neutral details. 


\section{MEMORY BIASES IN SOCIAL ANXIETY}

Supporting cognitive models of SA, which stipulate that the emergence of memory biases is likely to occur only under conditions in which threat appraisals are activated (Clark \& Wells, 1995; Rapee \& Heimberg, 1997), we observed a significant interaction between SA and condition across all three memory tasks, such that SA was related to memory only for social scenarios and not for non-social scenarios. Furthermore, across the memory tasks, higher levels of SA predicted worse memory performance when social scenarios were positively-valenced. Though the interaction between SIAS and condition predicting task 2 memory for positive scenario valence only approached significance, probing the interaction revealed that SIAS scores significantly predicted the recall of positive scenario outcomes. Contrary to our hypotheses, however, higher SA did not predict memory enhancements for social scenarios that were negatively-valenced for any of the tasks.

It may be useful to conceptualise these results within the context of research on positivity deficits in SA (Kashdan, 2007). Studies have shown that high SA individuals experience diminished positive affect and curiosity in response to social experiences (Kashdan \& Collins, 2010; Kashdan et al., 2009). Kashdan and colleagues have argued that positivity deficits might arise because social contexts prime socially anxious individuals' use of self-regulatory strategies geared toward self-protection and avoidance, which deplete the resources required for attending to and processing positive social cues (see Goodman, Doorley, \& Kashdan, 2018). Our findings suggest that poorer memory for positive social events among higher SA individuals may be another piece of this emerging puzzle.

These memory deficits for positive social scenarios did not extend to non-social situations, thus demonstrating a context-specific effect. Positive social information may be less memorable for higher SA individuals because such information is less coherent with the negative 


\section{MEMORY BIASES IN SOCIAL ANXIETY}

social self-schema that are held by individuals with high levels of SA (e.g., Conway, 2005;

Wilson \& Ross, 2003). These findings align with two recent studies that found high SA individuals' memory for positive feedback following a social task in the laboratory tended to erode over time in relation to non-anxious controls, with no differences in memory for neutral feedback emerging at either immediate or delayed recall (Glazier \& Alden, 2017, 2019).

Contrary to hypotheses, higher SA was generally not related to memory performance for negatively valenced social situations. One exception was for task 3 , in which higher SA was associated with poorer recognition of neutral details for both negative and positive social scenarios. It is unclear why this one memory task elicited degraded memory performance among higher SA individuals for social scenarios of any sort, particularly in light of our prediction that higher SA would predict superior memory for negative social scenarios only. It is possible that the cognitive demands of this particular task differed from those of the other two in ways that made high SA participants more vulnerable to the impact of interpretation bias or rumination. These cognitive processes commonly seen in high SA individuals have been found to affect memory accuracy among high SA participants for social information of any sort, irrespective of valence (e.g., Mansell \& Clark, 1999; Mellings \& Alden, 2000).

The present study was limited by its focus on the nature and quality of episodic nonautobiographical memories that did not require participants to engage in "mental time travel" to extract details from their own personal past. It is possible that mental time travel is a crucial component of memory biases for negative social information in SA, and that failure to support our hypothesis that higher SA would be associated with enhanced memory performance for negative social stimuli, as hasbeen shown for negative autobiographical memory details in 


\section{MEMORY BIASES IN SOCIAL ANXIETY}

previous studies (Moscovitch et al., 2018), may have been due to our focus on episodic memory for imagined scenarios rather than personally experienced social events.

Another potential limitation of the current study was that the ambiguous scenarios were only disambiguated in the final sentence; in other words, at a later stage of encoding than the neutral middle steps. Thus, encoding of these imagined scenarios may differ from real-life anxiety-provoking social situations in so far as the details of the former were not imbued with negative interpretation at the time they were encoded, whereas negative autobiographical events are always encoded in the presence of negative interpretation and the activation of high negative affect. Thus, it may be that negative interpretation and negative affect at the time of encoding are essential for inducing enhanced memory of such experiences. Future studies could extend our work by examining whether presenting a valenced cue that informs participants that the outcome will be positive or negative results in different types of memory biases if the cue is presented either before or after the processing and encoding of scenario details, or by comparing memory performance if scenarios are encoded while negative affect is high or low.

Finally, this study was conducted online, which limits our ability to draw conclusions about participants' engagement in the encoding task. We did not wish to interrupt participants' immersion in the task by embedding attention checks between scenarios, but in accordance with research on self-focused attention, it is possible that high SA participants for whom the social condition may have been particularly anxiety-provoking could have disengaged their attention from the task, thus impairing their recall on subsequent memory tasks. Our study design and measures do not permit any firm conclusions about the relations between memory performance, state arousal, and attentional engagement. Similarly, we cannot ascertain the degree to which participants immersed themselves when they were asked to imagine the social scenarios. It is 


\section{MEMORY BIASES IN SOCIAL ANXIETY}

possible that participants solely read the text prompts for the scenario displayed on scene, as opposed to engaging their imagination and vividly bringing to mind each of the scenarios.

While a strength of our paradigm is that it enabled us to verify the accuracy of participants' memories of scenarios, which is unfeasible in studies on autobiographical memory, future extensions of the current study could also examine the veracity of participants' memories for social and non-social scenarios by coding for intrusions and embellishments in their recall of these events; in so doing, a more fine-grained pattern of memory biases may emerge that would not be captured by solely scoring the amount of information recollected. Future studies using adaptations of this paradigm should also continue to explore the boundary conditions under which episodic memory biases occur in high SA individuals. In particular, research is needed to understand the causes and real-life consequences of memory performance deficits for positive social events for socially anxious individuals. If it can be established that the effects observed here might extend as well to individuals with a clinical diagnosis of SAD, future work must explore whether interventions can be designed to boost memory for positive social experiences and the potential benefits of such interventions for improving patients' lives.

\section{Acknowledgments}




\section{MEMORY BIASES IN SOCIAL ANXIETY}

This work was supported by the Social Sciences and Humanities Research Council of

Canada under grant [SSHRC Insight grant number: 435-2018-0959].

Declarations of conflicts of interest: none. 


\section{MEMORY BIASES IN SOCIAL ANXIETY}

\section{References}

American Psychiatric Association. (2013). Diagnostic and statistical manual of mental disorders (5th ed.). Washington, DC: Author.

Amin, N., Foa, E. B., \& Coles, M. E. (1998). Negative interpretation bias in social phobia. Behaviour Research and Therapy, 36, 945-957. doi:10.1016/S00057967(98)00060-6

Berna, C., Lang, T. J., Goodwin, G. M., \& Holmes, E. A. (2011). Developing a measure of interpretation bias for depressed mood: An ambiguous scenarios test. Personality and Individual Differences, 51, 349-354. doi:10.1016/j.paid.2011.04.005

Brown, T. A., Chorpita, B. F., \& Barlow, D. H. (1998). Structural relationships among dimensions of the DSM-IV anxiety and mood disorders and dimensions of negative affect, positive affect, and autonomic arousal. Journal of Abnormal Psychology, 107, 179.

Clark, D. M. (1999). Anxiety disorders: Why they persist and how to treat them. Behaviour Research and Therapy, 37, S5-S27. doi:10.1016/S0005-7967(99)00048-0

Clark, D. M., \& McManus, F. (2002). Information processing in social phobia. Biological Psychiatry, 51, 92-100. doi:10.1016/S0006-3223(01)01296-3

Clark, D. M., \& Wells, A. (1995). A cognitive model of social phobia. In R. G. Heimberg, M. Liebowitz, D. A. Hope, \& F. R. Schneider (Eds.). Social phobia: Diagnosis, assessment, and treatment (pp. 69-92). New York, NY: Guilford Press.

Conway, M. A. (2005). Memory and the self. Journal of Memory and Language, 53, 594-628. doi: $10.1016 /$ j.jml.2005.08.005

Eisner, L. R., Johnson, S. L., \& Carver, C. S. (2009). Positive affect regulation in anxiety disorders. Journal of Anxiety Disorders, 23, 645-649. doi:10.1016/j.janxdis.2009.02.001 


\section{MEMORY BIASES IN SOCIAL ANXIETY}

Glazier, B. L., \& Alden, L. E. (2017). Social anxiety and biased recall of positive information: It's not the content, it's the valence. Behavior Therapy, 48, 533-543. doi:10.1016/j.beth.2016.08.001

Glazier, B. L., \& Alden, L. E. (2019). Social anxiety disorder and memory for positive feedback. Journal of Abnormal Psychology, 128, 228-233. doi:10.1037/abn0000407

Goodman, F. R., Doorley, J. D., \& Kashdan, T. B. (2018). Well-being and psychopathology: A deep exploration into positive emotions, meaning and purpose in life, and social relationships. In E. Diener, S. Oishi, \& L. Tay (Eds.), Handbook of well-being. Salt Lake City, UT: DEF Publishers. DOI:nobascholar.com

Hackmann, A., Clark, D. M., \& McManus, F. (2000). Recurrent images and early memories in social phobia. Behaviour Research and Therapy, 38, 601-610. doi:10.1016/S00057967(99)00161-8

Hackmann, A., Surawy, C., \& Clark, D. M. (1998). Seeing yourself through others' eyes: A study of spontaneously occurring images in social phobia. Behavioural and Cognitive Psychotherapy, 26(1), 3-12.

Hertel, P. T., Brozovich, F., Joormann, J., \& Gotlib, I. H. (2008). Biases in interpretation and memory in generalized social phobia. Journal of Abnormal Psychology, 117, 278-288. doi:10.1037/0021-843X.117.2.278

Hirsch, C. R., \& Clark, D. M. (2004). Information-processing bias in social phobia. Clinical Psychology Review, 21, 741-770. doi:10.1016/j.cpr.2004.07.005

Hirsch, C. R., Clark, D. M., \& Mathews, A. (2006). Imagery and interpretations in social phobia: Support for the combined cognitive biases hypothesis. Behavior Therapy, 37, 223-236. doi: 10.1016/j.beth.2006.02.001 


\section{MEMORY BIASES IN SOCIAL ANXIETY}

Kashdan, T. B. (2007). Social anxiety spectrum and diminished positive experiences: Theoretical synthesis and meta-analysis. Clinical Psychology Review, 27(3), 348-365. doi:/10.1016/j.cpr.2006.12.003

Kashdan, T. B., \& Collins, R. L. (2010). Social anxiety and the experience of positive emotion and anger in everyday life: An ecological momentary assessment approach. Anxiety, Stress, \& Coping, 23(3), 259-272. doi:10.1080/10615800802641950

Kashdan, T. B., Morina, N., \& Priebe, S. (2009). Post-traumatic stress disorder, social anxiety disorder, and depression in survivors of the Kosovo War: Experiential avoidance as a contributor to distress and quality of life. Journal of Anxiety Disorders, 23(2), 185-196. doi:10.1016/j.janxdis.2008.06.006

Kashdan, T. B., \& Steger, M. F. (2006). Expanding the topography of social anxiety: An experience-sampling assessment of positive emotions, positive events, and emotion suppression. Psychological Science, 17, 120-128.

Kashdan, T. B., Weeks, J. W., \& Savostyanova, A. A. (2011). Whether, how, and when social anxiety shapes positive experiences and events: A self-regulatory framework and treatment implications. Clinical Psychology Review, 31, 786-799. doi:10.1016/j.cpr.2011.03.012

Koban, L., Schneider, R., Ashar, Y. K., Andrews-Hanna, J. R., Landy, L., Moscovitch, D. A., ... \& Arch, J. J. (2017). Social anxiety is characterized by biased learning about performance and the self. Emotion, 17, 1144. doi: 10.1037/emo00002961144

Krans, J., de Bree, J., \& Bryant, R. A. (2014). Autobiographical memory bias in social anxiety. Memory, 22, 890-897. doi: 10.1080/09658211.2013.844261 


\section{MEMORY BIASES IN SOCIAL ANXIETY}

Levine, L. J., Schmidt, S., Kang, H. S., \& Tinti, C. (2012). Remembering the silver lining:

Reappraisal and positive bias in memory for emotion. Cognition \& Emotion, 26, 871-884. doi:10.1080/02699931.2011.625403

Lovibond, P. F., \& Lovibond, S. H. (1995). The structure of negative emotional states:

Comparison of the Depression Anxiety Stress Scales (DASS) with the Beck Depression and Anxiety Inventories. Behaviour Research and Therapy, 33, 335-343. doi:10.1016/0005-7967(94)00075-U

Lundh, L. G., \& Öst, L. G. (1996). Recognition bias for critical faces in social phobics. Behaviour Research and Therapy, 34(10), 787-794. doi:10.1016/00057967(96)00035-6

Mansell, W., \& Clark, D. M. (1999). How do I appear to others? Social anxiety and processing of the observable self. Behaviour Research and Therapy, 37(5), 419-434. doi:10.1016/S0005-7967(98)00148-X

Marks, D. F. (1973). Visual imagery differences in the recall of pictures. British Journal of Psychology, 64(1), 17-24. doi:10.1111/j.2044-8295.1973.tb01322.x

Mathews, A., \& MacLeod, C. (2005). Cognitive vulnerability to emotional disorders. Annual Review of Clinical Psychology, 1, 167-195. doi:10.1146/annurev.clinpsy.1.102803.143916

Mattick, R. P., \& Clarke, J. C. (1998). Development and validation of measures of social phobia scrutiny fear and social interaction anxiety. Behaviour Research and Therapy, 36, 455470. doi:10.1016/S0005-7967(97)10031-6

Mellings, T. M., \& Alden, L. E. (2000). Cognitive processes in social anxiety: The effects of self-focus, rumination and anticipatory processing. Behaviour Research and Therapy, 38, 243-257. doi.org/10.1016/S0005-7967(99)00040-6 


\section{MEMORY BIASES IN SOCIAL ANXIETY}

Morgan, J. (2010). Autobiographical memory biases in social anxiety. Clinical Psychology Review, 30, 288-297. doi:10.1016/j.cpr.2009.12.003

Moscovitch, D. A., Vidovic, V., Lenton-Brym, A. P., Dupasquier, J. R., Barber, K. C., Hudd, T., ... \& Romano, M. (2018). Autobiographical memory retrieval and appraisal in social anxiety disorder. Behaviour Research and Therapy, 107, doi:10.1016/j.brat.2018.06.008

Rapee, R. M., \& Heimberg, R. G. (1997). A cognitive-behavioural model of anxiety in social phobia. Behaviour Research and Therapy, 35, 742-756. doi:10.1016/S00057967(97)00022-3

Reisberg, D., Pearson, D. G., \& Kosslyn, S. M. (2003). Intuitions and introspections about imagery: the role of imagery experience in shaping an investigator's theoretical views. Applied Cognitive Psychology, 17(2), 147-160. http://doi.org/10.1002/acp.858

Romano, M., Ma, R., Moscovitch, M., \& Moscovitch, D. A. (in press). J. S. Abramowitz and S. M. Blakey (Eds.) Clinical Handbook of Fear and Anxiety: Psychological Processes and Treatment Mechanisms, J. S. American Psychological Association.

Rubin, D. C. (Ed.). (1995). Remembering our past: Studies in autobiographical memory. New York, NY: Cambridge University Press. doi:10.1017/CBO9780511527913

Schacter, D. L., Guerin, S. A., \& Jacques, P. L. S. (2011). Memory distortion: An adaptive perspective. Trends in Cognitive Sciences, 15, 467-474. doi.org/10.1016/j.tics.2011.08.004

Wenzel, A., \& Cochran, C.K. (2006). Autobiographical memories prompted by automatic thoughts in panic disorder and social phobia. Cognitive Behavioral Therapy, 35, 129137. doi:10.1080/16506070600583130 


\section{MEMORY BIASES IN SOCIAL ANXIETY}

Wilson, A., \& Ross, M. (2003). The identity function of autobiographical memory: Time is on our side. Memory, 11, 137-149. doi:10.1080/741938210

Zlomuzica, A., Dere, D., Machulska, A., Adolph, D., Dere, E., \& Margraf, J. (2014). Episodic memories in anxiety disorders: clinical implications. Frontiers in Behavioral

Neuroscience, 8, 131. doi:10.3389/fnbeh.2014.00131 


\section{MEMORY BIASES IN SOCIAL ANXIETY}

Table 1. Characteristics of the study sample overall and in each condition separately

\begin{tabular}{|c|c|c|c|c|}
\hline & $\begin{array}{c}\text { Overall sample } \\
(n=197)\end{array}$ & $\begin{array}{c}\text { Social } \\
(n=98)\end{array}$ & $\begin{array}{c}\text { Non-Social } \\
(n=99)\end{array}$ & Test statistic \\
\hline Age in years: $M(S D)^{a}$ & $37.51(11.51)$ & $37.06(11.20)$ & $37.95(11.85)$ & $t_{(194)}=-0.54, p=.591$ \\
\hline Gender $(\%$ female $)$ & 61.4 & 60.2 & 62.6 & $\chi_{(4)}^{2}=3.19, p=.526$ \\
\hline Ethnicity ${ }^{b}$ & & & & $\chi_{(8)}^{2}=8.39, p=.396$ \\
\hline White/European & 75.1 & 70.4 & 79.8 & \\
\hline Black & 11.7 & 15.3 & 8.1 & \\
\hline Asian & 4.6 & 4.1 & 5.1 & \\
\hline Otherc & 8.1 & 9.2 & 7.0 & \\
\hline Education & & & & $\chi_{(5)}^{2}=7.01, p=.220$ \\
\hline High-school graduate & 16.8 & 14.3 & 19.2 & \\
\hline Some college/university education & 32.0 & 29.6 & 34.3 & \\
\hline Degree from college or university & 39.6 & 39.8 & 39.4 & \\
\hline Post-graduate degree & 10.7 & 15.3 & 6.1 & \\
\hline Employment status & & & & $\chi_{(6)}^{2}=4.23, p=.645$ \\
\hline $\begin{array}{l}\text { Employed full/part-time or self- } \\
\text { employed }\end{array}$ & 83.7 & 85.7 & 81.6 & \\
\hline Full-time home duties & 5.1 & 4.1 & 6.1 & \\
\hline Full-time student & 2.6 & 3.1 & 2.0 & \\
\hline Unemployed & 3.1 & 1.0 & 5.1 & \\
\hline Retired & 2.6 & 3.1 & 2.0 & \\
\hline Marital status & & & & $\chi_{(4)}^{2}=5.80, p=.215$ \\
\hline $\begin{array}{l}\text { Married/common law/engaged or } \\
\text { in committed relationship }\end{array}$ & 55.3 & 56.1 & 54.5 & \\
\hline Single & 34.5 & 31.6 & 37.4 & \\
\hline Divorced/separated & 7.6 & 11.2 & 4.0 & \\
\hline \multicolumn{5}{|l|}{ Self-report measures } \\
\hline SIAS & $27.39(16.16)$ & $26.66(16.27)$ & $28.13(16.11)$ & $t_{(193)}=-0.63, p=.527$ \\
\hline DASS-D & $8.55(10.35)$ & $8.22(10.55)$ & $8.87(10.19)$ & $t_{(195)}=-0.44, p=.663$ \\
\hline VVIQ-Short & $12.66(4.80)$ & $12.66(4.70)$ & $12.66(4.93)$ & $t_{(195)}=0.01, p=.992$ \\
\hline
\end{tabular}

Note. SIAS= Social Interaction Anxiety Scale; DASS-D = Depression subscale of the Depression, Anxiety and Stress Scale - Short Version; VVIQ-Short = Vividness of Visual Imagery Questionnaire ${ }^{\text {aAge }}$ missing for one person in social condition.

bEthnic groups are based on Canadian census categories.

c "Other" includes South Asian; Southeast Asian; Filipino; Indigenous/First nations; Hispanic;

"multiracial"; "biracial". 


\section{MEMORY BIASES IN SOCIAL ANXIETY}

Assignment and PreEncoding
243 participants recruited from MTurk

\section{Complete measures:}

Demographics questionnaire

Random assignment to scenario context condition

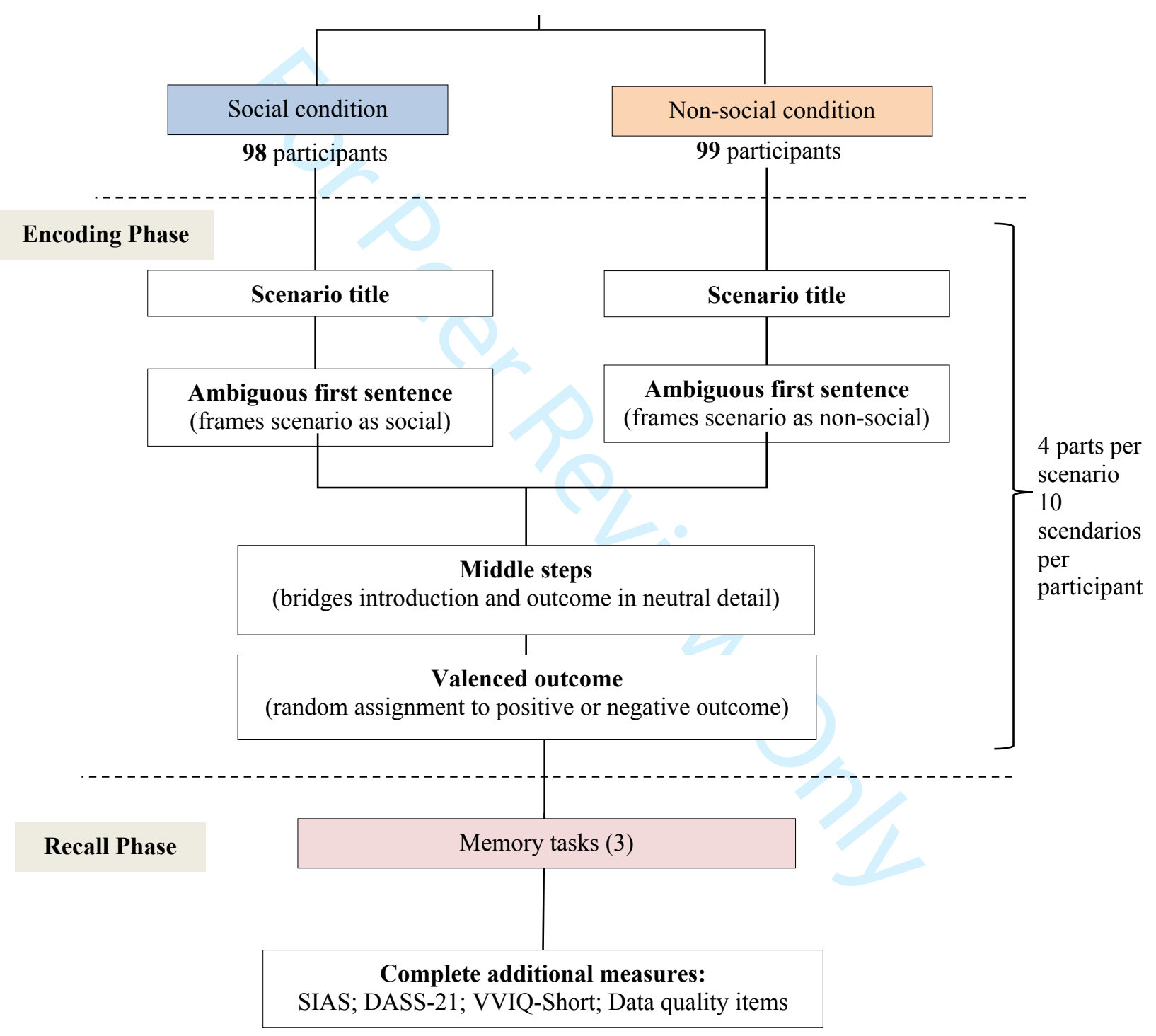

Figure 1. Study flow and procedure. 